\title{
Douglas E. Ott \\ The peritoneum and the pneumoperitoneum: a review to improve clinical outcome
}

Published online: 10 March 2004

(C) Springer-Verlag Berlin / Heidelberg 2004

\begin{abstract}
The pneumoperitoneum has a direct effect on the peritoneum and influences the outcome of laparoscopic surgery. The quality of the gas used, its temperature and dryness cause physical and chemical alterations that are preventable. The traditional gas is $20^{\circ} \mathrm{C}$ and $0.0002 \%$ relative humidity $(\mathrm{RH})$. The normal peritoneal cavity condition is $36^{\circ} \mathrm{C}$ and virtually $100 \% \mathrm{RH}$. Changing the gas to a more physiologic condition of $95^{\circ} \mathrm{C}$ and 95\% RH maintains peritoneal fluid viscosity and reduces hypothermia significantly by preventing evaporation, reduces inflammation, cell stress, and maintains peritoneal integrity by eliminating desiccation and reduces pain by preventing release of acute-phase reactive chemicals related to cell stress and disruption. Keeping peritoneal surfaces moist continuously during laparoscopy maintains a normal physiologic environment that is important to improving patient outcomes.
\end{abstract}

Keywords Peritoneum - Desiccation - Laparoscopy · Humidification · Pneumoperitoneum

\section{Introduction}

Maintaining homeostatic relationships of peritoneal cell physiology during surgical manipulation is important to reduce inflammation and its consequences, and to promote conditions for normal cellular repair and function. Once the peritoneal cavity is entered generalized tissue responses and defense mechanisms are set in motion. Repair and return to a condition of intact architecture is important for both form and function and is the goal of cellular mechanics, biology and biochemistry. The technical advancements of laparoscopic surgery have improved our understanding of surgical anatomy, shortened recovery and reduced pain and suffering. There is still

\section{E. Ott $(\bowtie)$}

Department of Biomedical Engineering, School of Engineering, Mercer University, 161 Gateway Drive, Macon, GA 31210, USA e-mail: gabiomed@mindspring.com

Tel.: +1-478-4716862 room for improvement. The water condition of the gaseous agents used to create the pneumoperitoneal dome has been ignored until recently. The two major contributors to a negative outcome related to the pneumoperitoneum are increased intra-abdominal pressure and the extreme dryness of the gas used and not to the specific chemical formula of the gas used for distention. Ceratis parabis elevated pressures of extremely dry gas for the creation and maintenance of a pneumoperitoneum, and whether it be carbon dioxide $\left(\mathrm{CO}_{2}\right)$, nitrous oxide $\left(\mathrm{N}_{2} \mathrm{O}\right)$, helium (He), argon (Ar) or air, all have and cause respiratory, homeostatic and physiologic consequences. Many of laparoscopic post-operative complaints are inextricably related to the very dry quality of the gas used throughout the procedure. There is a direct causeand-effect connection between the dryness of the gas used during laparoscopy and altered peritoneal cell function and stress and the reduction and improvement of symptoms by maintaining the normal physiologic homeostasis by conditioning the gas with heat and moisture. Keeping the gaseous environment of the peritoneal cavity during laparoscopy in its normal warm, wet state has cellular and clinical benefits and has created a new standard of care.

A significant cause of morbidity after surgery is intraabdominal adhesion formation [1]. As much as 33\% of patients who have had previous abdominal surgery are readmitted twice due to adhesion complication. Postoperative adhesions are associated with $40 \%$ of intestinal obstruction with $60-70 \%$ being of the small bowel $[2,3]$. When adhesions are present, they cause prolonged operating time and an increased complication rate. Pelvic adhesions distort normal anatomy and cause pain and infertility. There is no ideal method of preventing adhesion formation or reformation. Surgical technique is important to reducing the circumstances related to adhesion occurrence. Gentle tissue handling, preventing tissue desiccation and meticulous hemostasis, are attributes that prevent peritoneal damage and reaction that lead to adhesion formation. Because these factors are controllable, they can be dealt with to provide the best surgical environment to reduce the complications of ignoring 
them. Once the circumstance of peritoneal tissue stress and damage is present, there is a pathophysiologic path that leads to fibrin formation or fibrinolysis, adhesion formation or healing without adhesion formation [4].

Peritoneal desiccation is a constant surgical problem and when present is a requisite component associated with adhesion formation. Since the beginning of laparoscopy the gas has been cold and dry but unknown to surgeons, and the consequences of this intertwined with other symptoms. Cold dry gas laparoscopy is an improvement over cold dry laparotomy and warm wet laparoscopy is an improvement over cold dry or warm dry laparoscopy. There is a constant and universal plea to keep tissue conditions as close to normal as possible, to keep peritoneal surfaces moist, and to avoid damage since it provokes an inflammatory cellular response and complicates outcomes.

The normal condition of the peritoneal cavity is $36^{\circ}$ surfaces covered by a thin film of peritoneal fluid that is $100 \%$ relative humidity $(\mathrm{RH})$ in a steady state of fluid equilibrium and no exchange of gas from this potential space. The gas instilled for the pneumoperitoneum has less than 200 parts per million (ppm) water content $(0.0002 \% \mathrm{RH})$, is $20^{\circ} \mathrm{C}$, and the $3-1$ peritoneal cavity capacity (the average pneumoperitoneum volume) exchanged 20 or more times per hour with the gas flow reaching $37 \mathrm{~m} / \mathrm{s}[5,6,7,8]$. To think that putting a gas that is $16^{\circ} \mathrm{C}$ colder, delivered at high flow rates and having its volume exchanged rapidly, has little or no consequence is extremely incorrect and unwise. The rapid changes caused by this abnormal situation causes the intra-abdominal cavity and peritoneum to try to maintain physiologic equilibrium but results in stress and alters the peritoneum even before manual instrument manipulation or contact occurs. There are consequences to putting a cold, extremely dry gas into a confined space that is normally warm and wet. They are physical, thermal, chemical and biologic due to a desiccating effect. Other areas of the body that show detrimental effects from thinfilm desiccation are the corneal and the alveolar surfaces where dry gas similarly alters the thin-film surface active liquid and damages the thin tissue layer beneath.

The peritoneal fluid layer that is $60 \mu \mathrm{m}$ thin has a normal viscosity of 1.43 and a normal concentration of solutes and cells. The function of the fluid is to reduce friction between surfaces, to protect the peritoneal cell and to assist in healing if damage occurs serving as a first line of defense. Exposure to dry gas causes the peritoneal fluid to rapidly increase its viscosity, increase its solute content, to activate constituent cells and to act as a mediator for chemicals that are released from damaged cells [9]. The continuous uninterrupted undamaged moist peritoneum is essential to preventing adhesion formation. Adhesions are the result of a peritoneal tissue, chemical, and cellular response to trauma that disrupts the peritoneal cell, in the presence of blood. Peritoneal cell trauma can arise from any circumstance that causes cell damage. This can be from direct tactile touching by an instrument or mechanical touching with fingers.
Adjuncts have their place since trauma occurs beyond the known touching of tissues allowing coaptation of damaged surfaces that is a precursor for adhesion formation. These are treatments after trauma has occurred. Heating and hydrating the gas is a preventative measure before the desiccating trauma is inflicted. Prevention should be done prior to or in place of letting a problem occur or correcting it as a complication. Peritoneal desiccation and cell death is prevented by putting the proper amount of moisture into the gas $[10,11]$. Because of the mandated bone dry state of the gases used for a pneumoperitoneum, there are two types of desiccation effects suffered by the peritoneum, direct and indirect (collateral). As the gas flows through a needle or trocar it is collimated as a jet stream and impinges on whatever structure is in the gas path causing a depression of the fluid so that the gas comes in direct contact with the external surface of the peritoneal membrane. This is a direct desiccation effect. As the gas flows it causes a pressure defect of the thin $60-\mu \mathrm{m}$ film of peritoneal fluid on the tissue surface. This has two consequences:

1. The peritoneal fluid viscosity increases with the time and volume of gas exposure changing the viscosity and concentration of the peritoneal fluid prolonging contact time between tissue surfaces and changing the dynamics of tissue healing.

2. The contact of the dry gas with less than $200 \mathrm{ppm}$ water on the outside surface of the peritoneal cell membrane and the internal moisture content of $800,000 \mathrm{ppm}$ water causes a vapor pressure disequilibrium that results in peritoneal cell separation, loss of surface integrity, cell rupture, and structural damage to the underlying peritoneal connective tissue [11].

If these changes occur in the area to be excised, the damaged peritoneum is removed and a raw tissue area left. During the procedure gas insufflation that maintains abdominal distention is deflected from its directed impact site causing cumulative collateral indirect cell damage. Direct dry gas flow cell damage occurs with as little as $30 \mathrm{~s}$ exposure and indirect collateral peritoneal damage occurs with 8 min of gas flow [10,11].

The average gas volume used during laparoscopy is $60 \mathrm{l} / \mathrm{h}$ but often greatly exceeds this. Gas circulates and effects all parts of exposed surfaces within the abdominal dome as the pneumoperitoneum is maintained. The pneumoperitoneal space is maintained by pre-set pressure differential activation from the insufflator and is constantly replenished as gas is lost due to insensible leakage or intentional suction for removal of smoke, blood or irrigation. Regardless of the source of lost pressure, gas is constantly replaced by cold dry gas. It is the latent heat of vaporization that causes the thermal effect [12]. The very dry character of the gas causes desiccation and leads to peritoneal desertification [13, 14]. Indirect or collateral peritoneal damage results from the continuous intermittent gas exchange to maintain the pneumoperitoneal dome. The indirect effect on the peritoneal fluid and 
peritoneal cell is slower and cumulative dependent on total gas volume but just as important since the same desiccation changes occur and the desiccation changes are preventable.

The dryness of the gas, the effective diameter of the gas port exit within the abdomen, the total volume of gas used and length of exposure to the gas are all important factors causing the cumulative peritoneal effect. These same parameters of the cold dry gas flowing over intraabdominal structures and peritoneal surfaces causes constant heat and water vapor loss resulting in the clinical findings of $0.3^{\circ} \mathrm{C}$ temperature loss per hour of laparoscopy in addition to the normal heat loss during surgery and general anesthesia and is noted as an additional reason for the observed clinical hypothermia $[12,14,15$, $16,17]$. These consequences are eliminated or greatly reduced using warmed humidified gas $[12,14,15,16$, 17].

Preventing the effects of desiccation caused by cold dry or warm dry gas when used to create and maintain a pneumoperitoneum must be done. The peritoneal cavity is physiologically warm and wet. Exposing the peritoneal cavity to dry gas is not normal and creates a hostile condition that negatively alters cellular physiology and is the reason peritoneal surfaces even during laparoscopy should be kept constantly moist [18]. Prior to our knowledge about the dryness of the gas, its consequences were either unknown, unappreciated or considered the normal price to pay for a laparoscopic procedure. Laparoscopy since its inception has been done using nonphysiologic cold dry gas. Once we became aware of the gas condition it was necessary to admit the incorrect nature of the gas condition and alter it to be more normal and less damaging. Thinking that the altered condition of cold and dry in the normally warm wet space, and that it would have little to no effect, was naïve and presumptuous. Then warm dry gas was tried without success; an equally false presumption making the warm dry insufflation a blow dryer. It was and is still thought that whatever volume of gas it takes to complete a laparoscopic procedure, the $20^{\circ} \mathrm{C}, 0.0002 \% \mathrm{RH}$ gas in the $36^{\circ} \mathrm{C}, 95 \%+$ gas has little to no effect on the peritoneum or on clinical outcome. This is despite human clinical studies and animal model studies to the contrary.

Evaporation of a normally wet surface by extremely dry gas flowing onto a wet surface changes the ambient environmental conditions, accelerates evaporation, leads to loss of water content, dries, and eventually desiccates peritoneal cells. This clinical experiment of laparoscopy using cold dry gas has been practiced too long. It is time to re-establish and re-duplicate the peritoneal condition as closely as possible. Preventing peritoneal dehydration, maintaining peritoneal integrity, and reducing cellular damage has already been demonstrated.

Laparoscopy shows improvements that exceed laparotomy. Additional improvements for clinical outcomes have been shown using warm humidified gas. Animal model studies show less hypothermia, less peritoneal cell damage and maintenance of normal peritoneal fluid viscosity $[8,9,10,11,12,13,19,20,21]$. Compared with patients having laparoscopy using cold dry or warm dry gas, patients having warm humidified gas clinically show less hypothermia, less post-operative pain, shortened recovery room stay and less pain after leaving the hospital $[12,15,16,17,22,23,24,25,26]$.

\section{Peritoneum}

The embryologic derivation of the serous membranes, peritoneal, pleural, and pericardial cavities are the same and are lined by a layer of mesothelial cells. These mesothelial cells sit on a basement membrane overlying a submesothelial layer of extracellular matrix consisting of collagen, glycoproteins, proteoglycans and glycosaminoglycans. Deeper within this area are capillaries and lymphatics. The mesothelium and submesothelial stoma allow free diffusion and resorption of fluids. The external surface of peritoneal cells have microvilli and their intracellular volume is taken up mostly by the nucleus. Peritoneal cells can be detached from the basement membrane by trauma [4]. When a portion of the monolayer of peritoneal cells is damaged the normally tight intercellular junction is lost. When damaged the mesothelial cells secrete cytokines, interleukin (IL)-1, IL-6, and IL- 8 , tumor necrosis factor (TNF)- $\alpha$, transforming growth factor TGF)- $\beta$, intracellular adhesion molecule-1 (ICAM-1), hyaluronic acid, prostaglandin's, tissue plasminogen activator and plasminogen activator inhibitors (PAI) $[28,29,30]$. It has been reported that the amount of IL-6 and C reactive protein is at least four times less when warm humidified gas is used for a pneumoperitoneum than cold dry or warm dry gas demonstrating clinically that there is a decrease of acute-phase inflammatory response when a more homeostatic environment is maintained [31].

\section{Peritoneal fluid}

A thin film of peritoneal fluid of approximately $60 \mu \mathrm{m}$ covers the peritoneal cells. The peritoneal fluid acts to reduce friction between contacting surfaces and facilitates the normal function of the gastrointestinal tract, bladder, and the intra-peritoneal female genital tract. The lymphatic system allows peritoneal fluid to circulate with pleural fluid of the thoracic cavity and the vascular system. Molecules can travel into or out of the peritoneal cavity by transudation, exudation or facilitated transport, or by the lymphatics.

Cellular mediators produced by cellular components of the peritoneal fluid, poly-morphonucleated leukocytes, eosinophils, basophils, and macrophages, and the peritoneal cells play an active role in peritoneal healing. Because of the large peritoneal surface area, the potent activity of these mediators, the nature of peritoneal viscosity, concentration of its constituent and the ubiquity of the peritoneal fluid allows for an exaggerated response 
Fig. 1 Hematoxylin-eosin stain (magnification, $\times 200)$ of control peritoneum and peritoneum after 30-s exposure to cold dry or warmed humidified $\left(35^{\circ} \mathrm{C}\right.$ and $95 \%$ relative humidity) gas. No damage with warmed humidified gas and extensive damage with cold dry gas

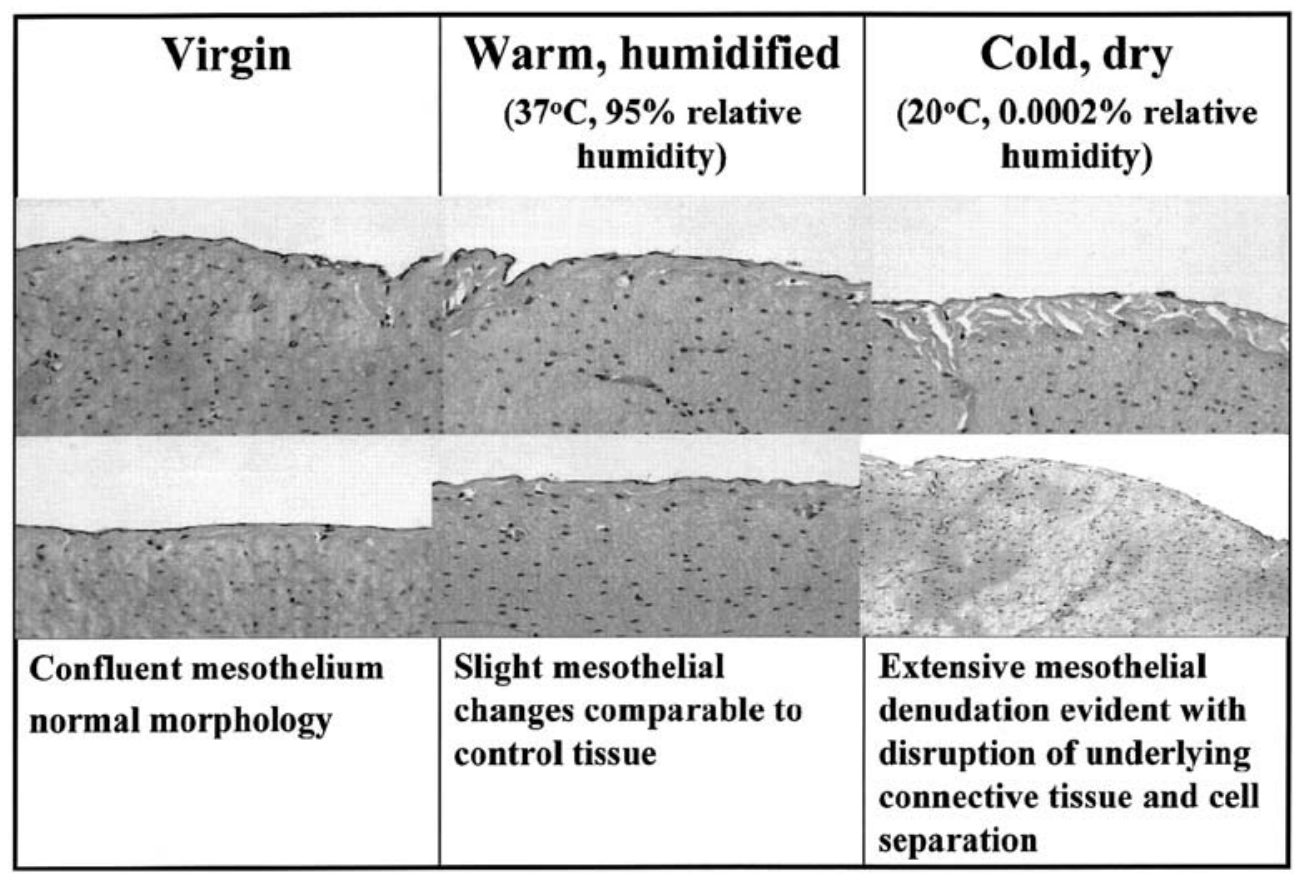

to irritating changes from normal homeostasis. In women the peritoneal fluid, its volume, and its cellular make-up is influenced by the menstrual cycle or hormonal concentrations and fluctuations. Disease conditions or pelvic pathology will also alter the peritoneal fluid character, like infection or endometriosis.

The changes of the cellular elements and the changes that occur to the peritoneum during healing are well understood for laparotomy circumstances. Desiccation is seen in as little as $8-10 \mathrm{~min}$ at laparotomy with room air exposure that is usually at 50\% RH [4]. Desiccation effects are seen at laparoscopy in as short a time as $30 \mathrm{~s}$ when cold dry or warm dry gas is used and little to no effect when $35^{\circ} \mathrm{C}$ and $95 \% \mathrm{RH}$ gas is used. The clinical, physiologic, and cellular effects of dry gas insufflation during laparoscopy have been accepted since the inception of laparoscopy, i.e., peritoneal desiccation, hypothermia, initiation of inflammation, and shoulder pain. These changes have been thought of as uncontrollable outcomes of laparoscopy. Laparoscopic surgery and the conditions under which it is performed is not the same as laparotomy. Laparotomy has 12-14 exchanges and turnover of operating room air that is usually $50 \% \mathrm{RH}$. Laparoscopy has at least 20 intra-abdominal exchanges of very dry gas based on a 3-1 pneumoperitoneum and 601 gas consumption per hour average. The gas enters the abdomen through trocars restricted by instruments or devices in their lumen creating an annular slot rather than the empty circle. This creates a nozzle effect and jet streaming of the gas due to the very reduced effective diameter of the gas exit site. Based on the diameter and rate of gas flow, gas speeds can reach $30 \mathrm{~m} / \mathrm{s}$ [7]. The gas stream touches and presses on whatever structure is in its path. The gas touch is not visible and has no tactile feedback to alert the surgeon to its occurrence. The gas stream eventually presses against the outside surface of tissue in its path causing a pressure effect defect creating a ring of peritoneal fluid. The edge of the peritoneal fluid ring rapidly loses water due to the difference of $200 \mathrm{ppm}$ compared with $995,000 \mathrm{ppm}$ or 1:4975 ratio and results in a physical defect of the peritoneal fluid covering the peritoneum.

While the peritoneal fluid is blown away, there is a loss of intra-cellular water caused by the dry gas creating a large vapor pressure differential and the intra-cellular water content attempts to equilibrate with the surrounding very dry extra-cellular cell gas. This attempt to maintain a water environment equilibrium between the surface of the external surface of the peritoneal cell membrane and the inner surface high internal intra-cellular water content stresses the membrane causing physical stress to the cell membrane changing its tensile strength, reducing its compliance, activating a chemical inflammatory stress response, desiccating the cell wall, and ultimately leading to cell rupture. The gas first forces the creation of a depression and separation of the peritoneal fluid allowing the gas stream to form a ring of peritoneal fluid with an area of no fluid in the central area. The gas in the depression is then in direct contact with the exposed external surface of the peritoneal cell membrane. The gas flow also causes loss of water from the rim of the peritoneal fluid forced away from the central area of the gas depression. When the gas is dry, evaporation of the water component of the peritoneal fluid occurs rapidly and with continuous exposure and increase in the peritoneal fluid viscosity changes from the normal 1.043 centipoise to as high as 108 [9]. Maintenance of peritoneal fluid viscosity is accomplished by having 95\% 
relative humidity water vapor in the gas stream. As a consequence of water loss from the peritoneal fluid there is a localized increase of its solutes making the fluid hypertonic and altering the normal ratios and proportions of peritoneal contents. These changes effect the normal hydraulic and lubricating benefit the fluid provides, i.e., they inhibit normal sliding and prolonging contact between tissue surfaces thus changing the dynamics of chemical exchange and increasing resident time of tissue contact. This occurs with $30 \mathrm{~s}$ exposure of constant dry gas and is prevented when the gas stream is $95 \% \mathrm{RH}$. As the gas stream is moved there is extension of the drying effect to other areas of the peritoneum in the gas path. Similar peritoneal fluid and cell wall changes occur due to indirect gas flow [10]. The continuous intermittent incremental stress and damage to the peritoneal fluid and peritoneal cells is cumulative leading to irreversible damage or prompts an apoptotic effect.

Pathologic evaluation by light microscopy and electron microscopy show there is gross damage to the peritoneal cell layer and the underlying connective tissue when cold dry gas is used and prevented when $36^{\circ} \mathrm{C}, 95 \%$ gas is used (Fig. 1) [4, 11, 19, 21]. Dry laparoscopic gas alters and damages the peritoneum, warm humidified gas does not [11].

\section{Conclusion}

It is known that peritoneal damage and disruption occurs due to drying and desiccation and is eliminated or greatly reduced when tissues are kept moist both at laparotomy and laparoscopy. This has led to the mantra "keep peritoneal tissues moist." It is also known that there is a cascade of chemical release occurring with peritoneal tissue stress, insult, and damage. During laparoscopy there is both direct and collateral damage to peritoneal surfaces when dry gas is used and is kept at a minimum when the gas stream is warm and moist. Virtually the totality of laparoscopic surgery has been performed and evaluated using a hostile abnormal non-physiologic environment by exposing and changing the peritoneal cavity to a dry gas wind tunnel. Changing the peritoneal condition to be more homeostatic, more physiologic, less hostile, less provocative, and less damaging improves outcomes.

The experiment of using cold dry gas for the pneumoperitoneum and changing the environment of the peritoneal cavity during laparoscopy has been practiced long enough. The normally luscious uninterrupted wet unstressed peritoneum is altered and damaged by circumstances promoting wind chill and desiccation creating a desert-like condition of the peritoneum. Peritoneum touched directly by the gas stream causes injury in the area of the impingement site and indirectly by deflected gas flow and volume turnover causing cumulative collateral peritoneal damage. The dry gas changes the peritoneal fluid, initiates an inflammatory response, and disrupts intact peritoneum. Warm humidified gas maintains the homeostatic condition of the peritoneal cavity while a pneumoperitoneum is present. It is time to end the assault on the peritoneum and condition the gas that creates and maintains the pneumoperitoneum and to re-create its normal physiologic friendly condition by warming and humidifying the gas.

\section{References}

1. Ellis H, Moran F, Thompson J et al. (1999) Adhesion-related hospital readmissions after abdominal and pelvic surgery: a retrospective cohort study. Lancet 353:1476-1480

2. Tulandi T (1986) Salpingo-ovariolysis: a comparison between laser surgery and electrosurgery. Fertil Steril 45:489-491

3. Menzies D (1993) Postoperative adhesions: their treatment and relevance in clinical practice. Ann R Coll Surg Engl 75:147153

4. Ryan G, Grobety J, Majno G (1973) Mesothelial injury and recovery. Am J Pathol 71:93-112

5. United States Pharmacopoeia and National Formulary and supplements (1984) XXI-NF

6. Ott DE (1991) Laparoscopic hypothermia. J Laparosc Surg $3: 127-131$

7. Lackey LW, Ott DE (2002) Terminal gas velocity during laparoscopy. J Am Assoc Gynecol Laparosc 9:297-305

8. Gray R, Ott D, Henderson A, Cochran S, Roth C (1999) Severe local hypothermia from laparoscopic gas evaporative jet cooling: a mechanism to explain clinical observations. JSLS $3: 171-177$

9. Ott D (2001) Laparoscopy and tribology: the effect of laparoscopic gas on peritoneal fluid. J Am Assoc Gynecol Laparosc $8: 117-123$

10. Ott D, Garner R, Wright E (2000) Unmodified carbon dioxide gas used during laparoscopy results in loss of peritoneal cell viability. JSLS 4:347

11. Wiseman D, Richardson J (2002) Humidity and temperature of insufflation gas on intact peritoneum. Am Assoc Gynecol Laparosc 9:S61; and 2003 PAX Sixth Symposum on Peritoneum, Amsterdam

12. Bessell J, Maddern G (1998) Influence of gas temperature during laparoscopic procedures. In: Rosenthal R, Friedman R, Philips E (eds) The pathophysiology of pneumo-peritoneum. Springer, Berlin Heidelberg New York, pp 18-27

13. Ott DE (2003) Desertification of the peritoneum by thin-film evaporation during laparoscopy. JSLS 7:189-195

14. Jacobs V, Morrison J, Mettler L, Mundhenke C, Jonat W (1999) Measurement of $\mathrm{CO}_{2}$ hypothermia during laparoscopy and pelviscopy: How cold it gets and how to prevent it. J Am Assoc Gynecol Laparosc 6:289-295

15. Ott D, Reich H, Love B et al. (1998) Reduction of laparoscopicinduced hypothermia, postoperative pain and recovery-room length of stay by pre-conditioning gas with the Insuflow device: a prospective randomized controlled multi-center study. JSLS 2:321-329

16. Kandaswamy R, Gillingham, Harmon J, Adolati M (2003) Decreased Incidence of hypothermia and peritoneal irritation in laparoscopic donor nephrectomy using a filter-hydrator-heating device (Insuflow). Surg Endosc 17:S230

17. Hamza M, Recart A, White P, Ogunnaike T, Schneider B (2003) Role of heated and humidified intraperitoneal gases using an Insuflow device during laparoscopic Roux-En-Y surgery: effect on temperature, post-operative pain and recovery. Anesthesiology 99:A618

18. Gomel V (1999) Foreward. In: DiZerega GS (ed) Peritoneal surgery. Springer, Berlin Heidelberg New York, pp vii-xi

19. Volz J, Koster S, Spacek Z, Paweletz N (1999) Characteristic alterations of the peritoneum after carbon dioxide pneumoperitoneum. Surg Endosc 13:611-614 
20. Volz J, Koster S, Weis M, Schmidt R et al. (1996) Pathophysiologic features of a pneumoperitoneum at lapaparoscopy: a swine model. Am J Obstet Gynecol:174:132-140

21. Hazebroek EJ, Schreve MA, Visser P, De Bruin RW, Marquet RL, Bonjer HJ (2002) Impact of temperature and humidity of carbon dioxide pneumoperitoneum on body temperature and peritoneal morphology. J Laparoendosc Adv Surg Tech A $12: 355-364$

22. Almeida O (2002) Awake microlaparoscopy with the Insuflow device. JSLS 6:199-201

23. Demco L (2001) Effect of heating and humidifying gas on patients undergoing awake laparoscopy. J Am Assoc Gynecol Laparosc 8:247-251

24. Mouton W, Bessell J, Otten K, Madern G (1999) Pain after laparoscopy. Surg Endosc 13:445-448

25. Farley D, Greenlee S, Larson D, Harrington J (in press) Double blind, prospective, randomized study of warmed, humidified $\mathrm{CO}_{2}$ insufflation versus standard $\mathrm{CO}_{2}$ for patients undergoing laparoscopic cholecystectomy. J Western Surg Assoc

26. Mouton W, Bessell J, Millard S, Baxter P, Maddern G (1999) A randomized controlled trial assessing the benefit of humidified insufflation gas during laparoscopic surgery. Surg Endosc 13:106-108

27. Douvdevani A, Raport J, Konforty A et al. (1994) Human peritoneal mesothelium synthesize IL-1, alpha and beta. Kidney Int 46:993-1001

28. Bachus K, Doty E, Haney A (1995) Differential effects of interleukin-1 alpha, tumor necrosis factor-alpha, indomethacin, hydrocortisone, and macrophage co-culture on the proliferation of human fibroblasts and peritoneal mesothelial cells. J Soc Gynecol Invest 2:636-642

29. Berborowicz A, Rodela H, Karon J et al. (1997) In vitro stimulation of the effect of peritoneal dialysis solution on mesothelial cells. Am J Kidney Dis 29:404-449

30. van Hinsburgh V, Kooistra T, Scheffer M et al. (1990) Characterization and fibrinolytic properties of human omental tissue mesothelial cells. Comparison with endothelial cells. Blood 75:1490-1497

31. Ott D (2003) Reduced peritoneal inflammation using wet gas compared to cold dry gas as measured by C-reactive protein and interleukin-6. JSLS 7:S27, 369 